\title{
Physiotherapy in Psychiatric Conditions
}

\section{Pradeep Thotekat*}

\section{B.P.T,M.P.T- Musculoskeletal Skeletal Disorder and Sports (Rajiv Gandhi University of Health Sciences, Karnataka, India)}

*Corresponding Author: Pradeep Thotekat, B.P.T,M.P.T- Musculoskeletal skeletal Disorder and Sports (Rajiv Gandhi University of Health Sciences, Karnataka, India)

Received: May 20, 2019; Published: June 13, 2019

DOI: 10.31080/ASOR.2019.02.0064

\section{Introduction}

Psychiatric disorders have traditionally been considered as mental rather than as physical illnesses. This is probably because they manifest with disordered functioning in the areas of emotion, perception, thinking and memory, and/or have had no clearly established biological basis.

Theoretical basis for physiotheraeutics in mental disorders

- Prior evidence of the cardiovascular benefits of exercise has shaped research into its psychological effects. The usual measure of fitness in psychological research has been aerobic fitness: the body's capacity for aerobic work.

- Physical exercise is enjoyable. Comparisons within studies have generally shown that mood has improved after strenuous exercise selectively in fitter or more active subjects. Opioid activation has been invoked to explain mood improvement.

- Morgan's (1969) demonstration that physically unfit psychiatric patients were more depressed than their fit counterparts.

- Explanations for psychological effects of exercise are many. Diverse suggestions have included changes in body temperature or cerebral blood flow, improvement in self-esteem, distraction from negative thoughts, or improved retrieval of positive thoughts, exercise as an exposure therapy, self mastery.

- Behavioral adaptation. Changes in several neurotransmitter systems have been causally implicated in behavioral adaptation.

\section{Central catecholamine systems in exercise}

Effects of exercise resemble those of other forms of stress. Brain norepinephrine turnover is increased by exercise. Effects of longterm exercise training also parallel repeated exposure to other stressors. Long-term regimes of swimming or running (whether compelled by a treadmill, or spontaneous) preserve or increase brain norepinephrine levels.

\section{Opioid mechanisms in effects of exercise}

Stress also activates central (and peripheral) opioid systems and this accounts for some instances of the analgesia which is caused by stress. Spontaneous exercise shares these effects, increasing endogenous opioid activity in the peripheral and central nervous system. There is indirect evidence that such release is psychologically important. Strenuous aerobic exercise is analgesic in man. The plasma beta-endorphin response to exercise increases with training, and there is evidence that the potentiation of opioid inhibition accounts for the reduction in cardiovascular stress responses after exercise training.

Stress adaptation is essentially a function of the repetition of exercise. Although repeated exposure to uncontrollable stressors eventually produces resistance to stress, exposure to controllable stress achieves this more quickly. The particular value of exercise might therefore be that it is a controllable stressor.

The potential value of physical exercise to the clinical psychologist derives not merely from its empirical and theoretical base, but from its popularity and face validity as a way of improving wellbeing. In this respect, for many individuals, it is likely to contrast with cognitive and behavioral approaches that are more common in the psychologist's armamentarium but appear less accessible to the general population.

\section{Classification of psychiatric disorders}

There are two main classifications of psychiatric disorders in current use:

- American Psychiatric Association's Diagnostic And Statistical Manual (4th edition), or DSM-IV

- World Health Organization's International Classification of Disease (10th edition), known as ICD-10.

Stress-related disorders

- $\quad$ Acute stress disorder

- Adjustment disorder

- $\quad$ Post-traumatic stress disorder 
Anxiety disorders

- Generalized anxiety

- Phobic anxiety

- $\quad$ Panic disorder

- Obsessive-compulsive disorder.

Affective (mood) disorders

- Depressive disorder

- Mania and bipolar disorder

Schizophrenia and delusional disorders

Substance misuse disorders

- Alcohol

- Drugs

Organic disorders

- $\quad$ Acute, e.g. delirium

- Chronic, e.g. dementia

Disorders of adult personality and behavior

- Personality disorder

- Factitious disorder

Eating disorders

- Anorexia nervosa

- Bulimia nervosa

Somatoform disorders

- $\quad$ Somatisation disorder

- Dissociative (conversion) disorder

- Pain disorder

- Hypochondriasis

- Body dysmorphic disorder

- Somatoform autonomic dysfunction

Neurasthenia and Puerperal mental disorders

Epidemology

In the general population, depression, anxiety disorders and adjustment disorders are most common (10\%) and psychosis is rare $(1-2 \%)$.

\section{Aetiology of psychiatric disorders}

Predisposing

- Increase susceptibility to psychiatric disorder

- Established in utero or in childhood

- Operate throughout patient's lifetime (e.g. genetic factors, congenital defects, chronic physical illness, disturbed family background)
Precipitating

- $\quad$ Trigger an episode of illness

- Determine its time of onset (e.g. stressful life events, acute physical illness)

Perpetuating

- Delay recovery from illness (e.g. lack of social support, chronic physical illness)

Diagnosing psychiatric disorders

A full psychiatric history and detailed mental state examination (MSE).

Presenting problems in psychiatric illness

Anxiety symptoms, Depressed mood, Elated mood, Delusions and hallucinations, Disturbed and aggressive behavior, Self-harm, Confusion, Alcohol misuse, Substance misuse, Medically unexplained somatic symptoms.

Treating psychiatric disorders

- Biological treatments

- $\quad$ Psychological treatments

- $\quad$ Social interventions

\section{Biological treatment}

These aim to relieve psychiatric illness by modifying brain function. Psychotropic drugs are widely used for various purposes. psychotropic drugs: Antipsychotic, Antidepressant, Mood-stabilizing, Anti-anxiety.

Electroconvulsive therapy (ECT) entails producing a convulsion by the administration of highvoltage, brief direct current impulses to the head while the patient is anaesthetized and paralyzed by muscle relaxants.

\section{Psychological treatments}

General or supportive psychotherapy

This should form part of all medical treatment. It involves empathic listening, in which the doctor encourages the patient to describe symptoms, express feelings and reflect on associated problems. The doctor should offer an explanation of the symptoms (and, where possible, a diagnosis), practical advice and such reassurance as is appropriate. For patients with incurable or chronic conditions, supportive psychotherapy may be the best treatment that can be offered.

\section{Cognitive therapy}

This is based on the observation that some psychiatric disorders are associated with systematic errors in the patient's conscious thinking: for example, a tendency to interpret events in a negative 
way or see them as unduly threatening. A triad of such cognitive errors has been described in depression. Cognitive therapy aims to help patients identify recurring negative thoughts and learn how to challenge them. It is widely used for depression, anxiety and bulimia nervosa, and increasingly in the management of somatoform disorders.

\section{Behavior therapy}

This is a practically orientated form of treatment, in which patients are helped to change their behavior.

In conditions such as phobic anxiety, working with the patient through a carefully constructed program of graded exposure to the feared stimulus is usually remarkably effective.

\section{Cognitive behavior therapy (CBT)}

This combines the methods of behavior therapy and cognitive therapy. It is the most widely available and most extensively researched psychological treatment.

\section{Problem-solving therapy}

This is a simplified brief form of CBT, which helps patients actively tackle problems in a structured way. It is of benefit in mild to moderate depression, and can be delivered by nonpsychiatric doctors and nurses after brief training.

\section{Psychodynamic psychotherapy}

This treatment, also known as 'interpretive psychotherapy', was pioneered by Freud, Jung and Klein, amongst others. It is based upon the theory that early life experience generates powerful motivating forces, of which the patient is unaware. Psychotherapy aims to help the patient to become aware of these unconscious factors on the assumption that, once identified, their negative effects are reduced. The relationship between therapist and patient is used as a therapeutic tool to identify issues in patients' relationships with others, particularly parents, which may be replicated or transferred to their relationship with the therapist. Explicit discussion of this transference is the basis for the treatment, which traditionally requires frequent sessions over a period of months or even years. Interpersonal therapy (IPT) is a specific form of brief psychotherapy which focuses on patients' current interpersonal relationships and is an effective treatment for mild to moderate depression.

\section{Social interventions}

Factors such as unemployment may not be readily amenable to intervention but others, but access to benefits and poor housing, are.
Patients can be helped to address these problems themselves by being taught a problem-solving approach. Befrienders and day centers can reduce social isolation, benefits advisers can ensure appropriate financial assistance, and medical recommendations can be made to local housing departments to help patients obtain more appropriate accommodation.

Psychiatric disorders and management

Stress-related disorders

- Acute stress reaction: Following a stressful event such as a serious medical diagnosis or a major accident, some people develop a characteristic pattern of symptoms. These include a sense of bewilderment, anxiety, anger, depression, altered activity and withdrawal. The symptoms are transient and usually resolve completely within a few days.

- Adjustment disorder: A more common psychological response to the onset or deterioration of a medical illness is a less severe but more prolonged emotional reaction. The predominant symptom is usually depression and/or anxiety, which is insufficiently persistent or intense to merit a diagnosis of depressive or anxiety disorder. There may also be anger, aggressive behavior and excessive alcohol use. Symptoms develop within a month of the onset of the stress, and their duration and severity reflect the course of the underlying physical condition, resolution tending to occur with physical recovery.

\section{Management and prognosis}

Ongoing contact with and support from a doctor or other who can listen, reassure, explain and advise are helpful and often all that is needed. Most patients do not require psychotropic medication, although benzodiazepines reduce arousal in acute stress reactions and can aid sleep in adjustment disorders. Psychotherapy may be helpful for some patients with abnormal grief reactions. These conditions usually resolve with time but can develop into depressive or anxiety disorders.

\section{Physiotherapist role}

- Encorage the patient to participate in exercise program. Exercise is found to lower emotional distress. Researches correlates perceived life stress with low levels of physical activity

- Patient can be given aerobic as well as anaerobic training. The effects of training found to reduce the cardiovascular changes during stress. 
- Focusing on responses to a specific stressor (being diagnosed HIV positive) La Perriere., et al. found that men who had trained aerobically for 5 weeks were protected from the increase in emotional distress and impairment of immune function (decline in natural killer cell number) shown by untrained controls

- An inhibitory effect of exercise on concurrent emotional stress responses is consistent with Physical Exercise and Stress Adaptation; that exercise can help one to cope with stress and other problems and with evidence, in animals, that wheelrunning exercise reduces sympathoadrenal or pituitary-adrenal responses to prior stress.

- $\quad$ Low-intensity training, which did not increase VO2 max, has more effectively reduced cardiovascular stress-responses than a high-intensity program which did improve fitness (Rogers., et al. 1996).

\section{Post-traumatic stress disorder (PTSD):}

This is a protracted response to a stressful event of an exceptionally threatening or catastrophic nature. Examples include natural disasters, terrorist activity, serious accidents and witnessing violent deaths. PTSD may also occur after distressing medical treatments.

Typical symptoms are recurrent intrusive memories (flashbacks) of the trauma, as well as sleep disturbance, especially nightmares (usually of the traumatic event) from which the patient awakes in a state of anxiety, symptoms of autonomic arousal, emotional blunting and avoidance of situations which evoke memories of the trauma. Anxiety and depression are associated and excessive use of alcohol or drugs frequently complicates the clinical picture.

\section{Management and prognosis}

The main aims are to provide support, direct advice and the opportunity for emotional catharsis. In established PTSD, structured psychological approaches (particularly cognitive therapy) and antidepressant medication are effective. There is some evidence that a novel therapy called eye movement desensitisation and reprocessing (EMDR) is effective. The condition runs a fluctuating course, with most patients recovering within 2 years. In a small proportion the symptoms become chronic.

\section{Physiotherapist Role}

- In relation to PTSD, involvement in exercise programs has been associated with lowering PTSD symptoms. In post-conflict settings, sport has been reported to improve confidence, body image, and to foster communication and attachment to positive role models. Sports and games may be able to alleviate symptoms of PTSD and improve mood and confidence. Sports and games may help build self-confidence, self discipline, body awareness, teamwork and communication skills (Meier 2005).

- One of the first studies to specifically evaluate the impact of aerobic exercise on PTSD involved a small sample of adults. These participants had experienced traumatic events such as tragic death of a relative or friend, sexual or physical assault, severe auto accident, combat, severe illness, injury, or disease.

Their exercise program consisted of 12 sessions in which there was a 10-minute warm up, 30 minutes of aerobic walking or jogging, and a 10-minute cool down period involving stretching and bicycling. Results of the study indicated that there were significant reductions in PTSD, anxiety, and depression following the aerobic exercise intervention and these reductions were maintained in a 1-month follow-up.

\section{Anxiety disorder}

These are characterised by the emotion of anxiety, worrisome thoughts, avoidance behaviour and the somatic symptoms of autonomic arousal. Anxiety disorders are divided into three main subtypes: phobic, paroxysmal (panic) and generalized.

- Phobic anxiety disorder: A phobia is an abnormal or excessive fear of an object or situation, which leads to avoidance of it (such as excessive fear of dying in an air crash leading to avoidance of flying). A generalized phobia of going out alone or being in crowded places is called agoraphobia. Phobic responses can develop to medical interventions such as venepuncture.

- Panic disorder: Panic disorder describes repeated attacks of severe anxiety, which are not restricted to any particular situation or circumstances. Somatic symptoms such as chest pain, palpitations and paraesthesiae in lips and fingers are common. The symptoms are in part due to involuntary overbreathing (hyperventilation). Patients often fear they are suffering from a serious illness such as a heart attack or stroke, and may therefore seek emergency medical attention. Panic disorder is often associated with agoraphobia.

- Generalised anxiety disorder: This is chronic anxiety associated with uncontrollable worry. Somatic symptoms of muscle tension and bowel disturbance often lead to a medical presentation

\section{Management of anxiety disorders Psychological treatment}

Explanation and reassurance are essential, especially when patients fear they have a serious medical condition. Specific treat- 
ment may be needed. Treatments include relaxation, graded exposure (desensitisation) to feared situations for phobic disorders, and CBT.

\section{Drug treatment}

Antidepressants are the drugs of choice. Benzodiazepines are useful in the short term but longterm use can lead to dependence. $\mathrm{A}$ b-blocker such as propranolol can help when somatic symptoms are prominent.

\section{Obsessive-compulsive disorder}

Obsessive-compulsive disorder (OCD) is characterized by obsessive thoughts, which are recurrent, unwanted and usually anxiety-provoking, and by compulsions, which are repeated acts performed to relieve feelings of tension. An example is repeated hand washing because of thoughts of contamination. The differential diagnosis is from normal checking behavior and from delusional beliefs about thought possession. OCD is equally common in men and women.

\section{Management and prognosis}

OCD usually responds to antidepressant drugs such as clomipramine, and to $\mathrm{CBT}$, which helps patients expose themselves to the feared thought or situation without performing the anxietyrelieving compulsions. Relapses are common and the condition often becomes chronic.

\section{Physiotherapy}

- The patients can be engaged in aerobic exercise as the studies found anxiolytic effects, exercise training can result in a moderate reduction in anxiety (Petruzzello., et al. 1991).

Note: In contrast, exercise may induce acute panic attacks (Broocks., et al. 1998; Barlow and Craske 1994) or increase subjective anxiety in patients with panic disorder more than in other people.

- In patients with high trait anxiety or generalized anxiety disorder, aerobic exercise training was superior to strength and mobility exercises (Steptoe., et al. 1989) or no treatment and comparable effective as cognitive behavior therapy (McEntee and Halgin 1999).

- Case reports (Dractu 2001) and two published clinical studies suggest that exercise training may be used therapeutically in patients with anxiety neurosis (Sexton., et al. 1989) and panic disorder (Broocks., et al. 1998).

- Strategies used with cognitive behavioral therapy may be applied to exercise (training): situational analysis, goal setting, self monitoring, homework activities, and sup- portive follow-up may support compliance and maintain a new behavior (Otto., et al. 2007).

- Theoretical rationales for why exercise might work to reduce anxiety symptoms include the monoamine neurotransmitter theory, exercise as a buffer to stress, exercise as a form of meditation, and exercise as a form of exposure therapy (Weyerer and Kupfer, 1994).

\section{Mood or affective disorders include}

- Unipolar Depression: One or more episodes of low mood and associated symptoms

- Bipolar Disorder: Episodes of elevated mood interspersed with episodes of depression

- Dysthymia: Chronic low-grade depressed mood without sufficient other symptoms to count as 'clinically significant' or 'major' depression.

\section{Depression}

It is a major cause of disability and of suicide.

If co morbid with a medical condition, depression magnifies disability, diminishes adherence to medical treatment and rehabilitation, and may even shorten life expectancy. Such co morbid depression may incrementally worsen health more than any combination of chronic diseases without depression.

\section{Aetiology}

There is a genetic predisposition to depression, especially when of early onset. The number and identity of the genes are largely unknown but the serotonin transporter gene is a candidate. Adversity and emotional deprivation early in life also predispose to depression. Depressive episodes are often triggered by stressful life events (especially those that involve loss), including medical illnesses. Associated biological factors include hypofunction of monoamine neurotransmitter systems (5-HT and noradrenaline (norepinephrine)) and abnormal hypothalamo-pituitary-adrenal axis (HPA) regulation, which results in elevated cortisol levels that do not suppress with dexamethasone.

\section{Symptoms}

Psychological

- $\quad$ Depressed mood

- $\quad$ Reduced self-esteem

- Pessimism

- $\quad$ Guilt

- $\quad$ Loss of interest

- $\quad$ Loss of enjoyment (anhedonia)

- $\quad$ Suicidal thinking 
Somatic

- $\quad$ Reduced appetite

- Weight change

- Disturbed sleep

- Fatigue

- $\quad$ Loss of libido

- Bowel disturbance

- Motor retardation (slowing of activity)

\section{Management and prognosis}

There is evidence that both drug and psychological treatments work in depression. In practice, the choice is determined by patient preference and local availability. Severe depression complicated by psychosis, dehydration or suicide risk may require ECT.

\section{Psychological treatments}

Both CBT and interpersonal psychotherapy are as effective as antidepressants for mild to moderate depression. Antidepressant drugs are, however, preferred for severe depression. Drug and psychological treatments can be used in combination. The risk of suicide in an individual who has had a depressive disorder is ten times greater than in the general population.

\section{Bipolar disorder}

Bipolar disorder is an episodic disturbance with interspersed periods of depressed and elevated mood; the latter is known as hypomania, or mania when severe. The lifetime risk of developing bipolar disorder is approximately $1 \%$. Onset is usually in the teens, and men and women are equally affected. Bipolar disorder has been divided into two types:

- Bipolar I disorder has a clinical course characterized by one or more manic episodes or mixed episodes. Often in dividuals have also had one or more major depressive episodes.

- $\quad$ Bipolar II disorder features depressive episodes which are more frequent and more intense than manic epi sodes, but there is a history of at least one hypomanic episode.

\section{Aetiology}

Bipolar disorder is strongly heritable $(80 \%)$. Relatives of patients have an increased incidence of both bipolar and unipolar affective disorder but life events, such as physical illness and medication, may play a role in triggering episodes.

\section{Diagnosis}

Isolated episodes of hypomania or mania do occur but they are usually preceded or followed by an episode of depression. Psycho- sis may occur in both the depressive and the manic phases, with delusions and hallucinations that are usually in keeping with the mood disturbance. This is described as an affective psychosis. Patients who present with symptoms of both bipolar disorder and schizophrenia may be given a diagnosis of schizoaffective disorder.

\section{Management and prognosis}

Depression should be treated, as described above. Manic episodes usually respond well to antipsychotic drugs. Prophylaxis to prevent recurrent episodes is important. The main drugs used are lithium, carbamazepine and sodium valproate. Lamotrigine and quetiapine are increasingly being used. Caution must be exercised when stopping these, as a relapse may follow.

\section{Physiotherapy}

1.Research indicates that significant improvements can be achieved with clinically depressed individuals, with as little as $\mathbf{5}$ weeks of thrice weekly supervised sessions of aerobic (walk or run) or nonaerobic exercise activity of low to moderate intensity (50\% of maximum heart rate) lasting from 20-60 min in duration.

In addition, follow-up assessments have indicated that treatment gains can be maintained for up to 1 year, particularly if some level of regular activity is continued (Doyne., et al. 1987; Greist., et al. 1979). Most notably, exercise therapy has proven to be four to five times more cost-effective than traditional treatments for depression (Freemont and Craighead, 1987; Greist., et al. 1979).

The study of Dimeo., et al. (2001) suggests that in treatmentresistant patients with major depression, 30 min of treadmill walking for ten consecutive days may be sufficient to produce a clinically relevant and statistically significant reduction in depression, as measured with the Hamilton Depression rating Scale. These findings are substantiated by a more recent study involving a placebo exercise group (lowintensity stretching and relaxation exercises) in patients receiving a standard antidepressant treatment: the reduction of depression scores and the response rates were larger in the exercise training group Knubben., et al. 2006.

Blumenthal., et al. (1999) could show that 16 weeks of group exercise training in older patients with major depression was as effective as antidepressant treatment with sertraline.

\section{Schizophrenia}

Schizophrenia is a psychosis characterised by delusions, hallucinations and lack of insight. Acute schizophrenia may present with disturbed behaviour, marked delusions, hallucinations and disordered thinking, or with insidious social withdrawal and less obvious delusions and hallucinations. 


\section{Aetiology}

There is a strong genetic contribution, probably involving many susceptibility genes, each of small effect. Environmental risk factors include obstetric complications and urban birth.

Brain imaging techniques have identified subtle structural abnormalities, including an enlargement of the lateral ventricles and an overall decrease in brain size (by about $3 \%$ on average),with relatively greater reduction in temporal lobe volume (5-10\%).

Episodes of acute schizophrenia may be precipitated by social stress and cannabis, which increase dopamine turnover and sensitivity. Consequently, schizophrenia is now viewed as a neurodevelopmental disorder, caused by abnormalities of brain development associated with genetic predisposition and early environmental influences, but precipitated by later triggers.

\section{First-rank symptoms of acute schizophrenia}

- $\mathrm{A}=$ Auditory hallucinations - second or third person

- $\mathrm{B}=$ Broadcasting, insertion/withdrawal of thoughts

- $\quad \mathrm{C}=$ Controlled feelings, impulses or acts ('passivity' experi ences/phenomena)

- $\quad \mathrm{D}=$ Delusional perception (a particular experience is bi zarrely interpreted)

Symptoms of chronic schizophrenia (negative symptoms)

- Flattened (blunted) affect

- Apathy and loss of drive (avolition)

- Social isolation

- Poverty of speech

- Poor self-care

\section{Management}

First-episode schizophrenia usually requires admission to hospital because patients lack insight that they are ill and are unwilling to accept treatment. In some cases, they may be at risk of harming themselves or others. Subsequent acute relapses and chronic schizophrenia are now usually managed in the community.

\section{Drugs}

Antipsychotic agents are used. These may be divided into conventional (typical, first-generation) drugs such as chlorpromazine and haloperidol, and newer or atypical (also so-called novel or second-generation) drugs such as clozapine. All are believed to work by blocking D 2 dopamine receptors in the brain. Patients who have not responded to conventional drugs may respond to newer agents, which are also less likely to produce unwanted extrapyramidal side-effects but do tend to cause greater weight gain.

\section{Psychological treatment}

Psychological treatment, including general support for the patient and his or her family, is now seen as an essential component of the therapeutic plan. CBT may help patients to cope with treatment-resistant symptoms. There is evidence that personal and/or family education reduces the rate of relapse.

\section{Social treatment}

After an acute episode of schizophrenia has been controlled by drug therapy, social rehabilitation may be required. Recurrent illness is likely to cause disruption to patients' relationships and their ability to manage their accommodation and occupation; consequently, they may need help to obtain housing and employment.

A graded return to employment and sometimes a period of supported accommodation are required.

\section{Physiotherapy}

- $\quad$ Physical inactivity and poor cardiorespiratory fitness could also explain brain volume reductions seen in schizophrenia. If physical inactivity and poor cardiorespiratory fitness explain the brain volume reductions in schizophrenia, one would expect that the brain volume decreases would diminish when cardiorespiratory fitness increases. Interestingly, animal studies have unequivocally shown that physical exercise positively affects brain morphology, especially in the hippocampus, and brain functioning. In healthy elderly, studies have shown that exercise increases cerebral grey and white matter and hippocampal volumes.

- Improvement in cardiorespiratory fitness is associated with cortical thickening (or less thinning)in the left hemisphere in patients with schizophrenia as well as in healthy controls. This suggests that moderate exercise induces subtle changes in cerebral(grey matter)volume most clearly(at least measurably) expressed in changes in cortical thickness. The underlying mechanisms of brain volume increases as a result of improved fitness are still unknown, but increased production of neurotrophic growth factors, improved vascularisation, and improved energy metabolism, all of which are of central importance in neurogenesis (Cotman andBerchtold,2002; van Praag, 2008, 2009) seem to play a role. Given the crucial role exercise plays in neuronal plasticity, exercise therapy may ameliorate brain abnormalities in schizophrenia.

Alcohol misuse and dependence

Alcohol consumption associated with social, psychological and physical problems constitutes harmful use. 
- Aetiology: Availability of alcohol and social patterns of use appear to be the most important factors. Genetic factors may play some part in predisposition to dependence. The majority of alcoholics do not have an associated psychiat ric illness, but a few drink heavily in an attempt to relieve anxiety or depression.

- Diagnosis: Alcohol misuse may emerge during the pa tient's history, although patients may minimize their in take. It may also present via its effects on one or more aspects of the patient's life, listed below. Alcohol admitted dependence commonly presents with withdrawal in those to hospital, as they can no longer maintain their high alcohol intake in this setting.

\section{Complications of chronic alcohol misuse}

Social problems include absenteeism from work, unemployment, marital tensions, child abuse, financial difficulties and problems with the law, such as violence and traffic offences.

Psychological problems

- Depression is common Alcohol has a direct depressant effect and heavy drinking creates numerous social problems. At tempted suicide and completed suicide are often associated with alcohol misuse.

- Anxiety is relieved by alcohol. People who are socially anx ious may consequently use alcohol in this way and may de velop dependence. Conversely, alcohol withdrawal increases anxiety.

- Alcoholic hallucinosis is a rare condition in which alcoholic individuals experience auditory hallucination in clear con sciousness.

- Alcohol withdrawal: Symptoms usually become maximal about 2 days after the last drink and can include seizures ('rum fits').

- Delirium tremens is a form of delirium associated with se vere alcohol withdrawal. It has a significant mortality and morbidity.

\section{Effects on the brain}

The familiar features of drunkenness are ataxia, slurred speech, emotional incontinence and aggression. Very heavy drinkers may experience periods of amnesia for events which occurred during bouts of intoxication, termed 'alcoholic blackouts'. Established alcoholism may lead to alcoholic dementia, a global cognitive impairment resembling Alzheimer's disease, but which does not progress and may even improve if the patient becomes abstinent. Indirect effects on behavior can result from head injury, hypoglycemia and encephalopathy.

\section{Management and prognosis}

The provision of clear information from a doctor about the harmful effects of alcohol and safe levels of consumption is often all that is needed. In more serious cases, patients may have to be advised to alter leisure activities or change jobs if these are contributing to the problem. Psychological treatment is used for patients who have, If alcohol dependence is suspected, withdrawal syndromes can be prevented, or treated once established, with benzodiazepines.

\section{Substance misuse disorder}

Dependence on and misuse of both illegal and prescribed drugs is a major problem world-wide.

\section{Sedatives}

These commonly give rise to physical dependence, the manifestations being tolerance and a withdrawal syndrome. They include benzodiazepines, opiates (including morphine, heroin, methadone and dihydrocodeine) and barbiturates (now rarely prescribed). Overdosage can be dangerous with the opiates and benzodiazepines, primarily as a result of respiratory depression. Withdrawal from opiates is notoriously unpleasant, and withdrawal from benzodiazepines and barbiturates may be dangerous because of seizures. The withdrawal syndrome, which can start within 12 hours of the drug's last use, presents with intense craving, rhinorrhoea, lacrimation, yawning, perspiration, shivering, piloerection, vomiting, diarrhoea and abdominal cramps.

Examination reveals tachycardia, hypertension, mydriasis and facial flushing.

\section{Stimulants}

These include amphetamines and cocaine. They are less dangerous than the sedatives in overdose, although they can cause cardiac and cerebrovascular problems through their pressor effects. With prolonged heavy use, psychiatric disturbance can be prominent. Physical dependence syndromes do not arise, but withdrawal causes a rebound lowering in mood and can give rise to an intense craving for further use. Chronic ingestion can cause a syndrome similar to schizophrenia. A toxic psychosis can occur with high levels of consumption, and tactile hallucinations (formication) may be prominent.

\section{Hallucinogens}

The hallucinogens are a disparate group of drugs that cause prominent sensory disturbances. They include cannabis, ecstasy, lysergic acid diethylamide (LSD) and Psilocybin (magic mushrooms). A toxic confusional state can occur after heavy cannabis consumption. Acute psychotic episodes are well recognised, espe- 
cially in those with a family or personal history of psychotic illness, and there is evidence that prolonged heavy use increases the risk of developing schizophrenia. Paranoid psychoses have been reported in association with ecstasy.

\section{Organic solvents}

Solvent inhalation (glue sniffing) is popular in some adolescent groups. Solvents produce acute intoxication characterised by euphoria, excitement, dizziness and a floating sensation. Further inhalation leads to loss of consciousness; death can occur from the direct toxic effect of the solvent, or from asphyxiation if the substance is inhaled from a plastic bag.

\section{Aetiology}

Many of the aetiological factors for alcohol misuse also apply to drug dependence. The main factors are cultural pressures, particularly within a peer group, and availability of a drug. In the case of some drugs, medical overprescribing has increased their availability, but there has also been a relative decline in the price of illegal drugs. Most drug users take a range of drugs-socalled polydrug misuse.

\section{Diagnosis}

As with alcohol, the diagnosis either may be apparent from the history, or may only be made once the patient presents with a complication. Drug screening of samples of urine or blood can be very valuable in confirming the diagnosis, especially if the patient persists in denial.

\section{Physiotherapy}

1. Spontaneous remission rates from substance use disorders very low but relapse rates are high ranging from 60 to $90 \%$ within the first year following treatment. Therefore, the development of interventions to decrease relapse rates and maintain treatment gains for substance abusing patients is critically important.

2. Relapse prevention models have included lifestyle modifications as a potentially important component of recovery (Marlatt and Donovan, 2005). For example, Larimer, Palmer, and Marlatt (1999) describe the importance of helping the client develop "positive addictions" such as increased physical activity.

3. It has been argued that the benefits of exercise in early recovery may include increased selfefficacy for abstinence, improved mood states, decreased urges and cravings, and could function as a useful coping strategy (Read and Brown, 2003).
4. Moderate-intensity exercise was found to provide short-term relief from urges to drink alcohol (Ussher, Sampuran, Doshi, West, and Drummond, 2004).

5. Low rates of exercise involvement prior to substance abuse treatment, suggests that during the height of their addiction, when they were actively using alcohol and/or drugs, they may not have been able to prioritize or follow-through with an exercise program. However, given even a brief period of sobriety, participants were able to recognize and be interested in incorporating regular physical activity into their lives and as a potentially important part of their recovery

6. walking and brief bouts of physical activity have also been associated with decreased urges to drink or smoke cigarettes (Taylor, Ussher and Faulkner, 2007;Ussher., et al. 2004). These findings for walking interventions are comparable to those demonstrated with more structured, supervised exercise programs. Therefore, a walking intervention may be an effective approach for increasing physical activity and increasing physical and psychological health benefits among substance abusing patients in early recovery

7. Pedometers have been used to improve self-monitoring, facilitate goal setting, and enhance motivation in numerous exercise intervention studies. The inclusion of pedometers in an exercise intervention for substance abusing patients may play an important role in increasing participant motivation and increase self-monitoring. In addition, the use of monetary incentives has been utilized in previous exercise intervention studies as a means of increasing motivation and adherence.

\section{Delirium, dementia and organic disorders}

Delirium, dementia and other organic disorders can be thought of as medical conditions rather than psychiatric disorders, but are included in psychiatric classifications and sometimes misdiagnosed because they manifest with disturbed behaviour.

\section{Delirium}

Delirium is common in acute medical settings, affecting more than half of patients in highdependency and intensive care units. Delirium (also known as acute confusional state) is a disorder of consciousness and attention combined with abnormalities of cognition and perception.

\section{Physiotherapy management}

1. Exercise therapy may lower the effective required dose of antipsychotic drugs in patients with delirium.

2. The administered dose of antipsychotic drugs was significantly lower in the Exercise group versus the Non exercise group. 
Thus, symptoms of delirium could be attenuated in patients who concurrently receive exercise therapy upon delirium occurrence. Inouye., et al. [14] found that a multi component intervention prevented the development of delirium

3. Patients may have improved their temporospatial orientation to some extent with exercise therapy, effectively reducing one complicating factor for delirium. Centeno., et al. advised that patients should be surrounded by familiar people and objects and that caretakers should use extensive physical contact. In addition, regular exercise therapy supervised by the physical therapist in charge may improve patient daytime rhythms and support their orientation. It also seems plausible that exercise therapy increases daytime activity by preventing extended immobility and encouraging normal sleep-wake cycles.

4. Increased daytime activity due to preventing extended immobility and encouraging normal sleep-wake cycles may play a valuable role in delirium management.

\section{Dementia}

It is defined as a global impairment of cognitive function, and although memory is most affected in the early stages, deficits in visuo-spatial function, language ability, concentration and attention gradually become apparent.

\section{Medical management and prognosis}

The anticholinesterase inhibitors, such as donepezil, may arrest progression for a time in Alzheimer-type dementia, while addressing the underlying vascular risk factors may slow deterioration in vascular dementia, but neither can be reversed. Psychotropic drugs may help where there is associated disturbance of sleep, perception or mood, but should only be used with care, especially since evidence shows increased mortality in patients treated long term with these agents.

\section{Physiotherapy}

Systematic review by Blankevoort and colleagues including 16 clinical trials on physical activity interventions in patients with dementia, concluded that physical activity can improve physical functioning at almost every stage of dementia.

They reported that a combination of different types of physical activity (e.g. endurance, strength and balance training) led to greater improvement of physical function than progressive resistance training alone, suggesting that amulti-component physical activity intervention may be more effective.

In a recent review paper Lazarov., et al. investigated whether neurogenesis enhancing activities could counteract age-related cognitive impairment and symptomatology. Lazarov., et al. pointed out that age-dependent reduction of neurogenesis can be minimized and even better can be partially reversed when rodents have the opportunity to exercise

The American College of Sports Medicine (ACSM) and the American Heart Association have reviewed the evidence for the health benefits of physical activity and based on this evidence they have developed recommendations for physical activity for older adults.

The recommendation is for older adults to do moderate-intensity aerobic physical activity for a minimum of 30 min on 5 days each week or vigorous- intensity aerobic activity for a minimum of 20 min on 3 days each week.

In addition, they should include muscle strengthening, flexibility and balance exercises. The ACSM recommendations also highlight the need for older adults to have an activity plan that integrates preventative and therapy recommendations.

\section{Eating disorders}

There are two well-defined eating disorders, anorexia nervosa (AN) and bulimia nervosa (BN), which share some overlapping features.

\section{Anorexia nervosa}

There is marked weight loss, arising from food avoidance, often in combination with bingeing, purging, excessive exercise, or the use of diuretics and laxatives.

Body image is profoundly disturbed so that, despite emaciation, patients still feel overweight and are terrified of weight gain. These preoccupations are intense and pervasive, and the false beliefs may be held with a conviction approaching the delusional. Anxiety and depressive symptoms are common accompaniments.

\section{Diagnostic criteria}

- Weight loss of at least $15 \%$ of total body weight (or body mass index $\leq 17.5$ )

- $\quad$ Avoidance of high-calorie foods

- Distortion of body image so that patients regard themselves as fat even when grossly underweight

- $\quad$ Amenorrhea for at least 3 months

\section{Bulimia nervosa}

In $\mathrm{BN}$, patients are usually at or near normal weight (unlike in AN), but display a morbid fear of fatness associated with disordered eating behavior.

They recurrently embark on eating binges, often followed by corrective measures such as selfinduced vomiting. The prevalence 
is similar to or slightly greater than that of AN, but only a small proportion of sufferers reach treatment services.

\section{Management and prognosis}

CBT achieves short- and long-term improvements. Guided selfhelp and interpersonal psychotherapy may also be of value. There is also evidence for benefit from the SSRI, fluoxetine, although high doses (60 mg daily) and long courses (1 year) may be required; this appears to be independent of the antidepressant effect.

\section{Physiotherapist Role}

Exercise results in improvements in the malleable ED risk and maintenance factors of self-esteem, anxiety, depression, negative mood, and body image. Exercise results in improved social bonds and relations. Because patients with ED may have disturbed social relations (e.g. isolated eating and reduced social contact, exercise may aid in improving social behaviors. In short, theoretical justification suggests that by improving physical fitness through regular healthy exercise, patients with ED may experience improved selfesteem, body image, and mood, as well as a reduction in the uncomfortable sensations of bloating and distention during eating. Additionally, exercise promotes self-regulation. Therefore, exercise may reduce bodily tensions and negative mood and increase tolerance to everyday stress, which are all triggers for binging and purging.

A reciprocal relationship occurs whereby exercise results in improvements in well-being, and improved well-being results in increased exercise. Similarly, a reciprocal relationship occurs whereby improved well-being results in decreased ED risk factors, maintenance factors, and outcomes, which ultimately results in decreased ED prevalence. Exercise during ED treatment may improve many biopsychosocial outcomes. For example, Sundgot-Borgen., et al. found that an exercise program resulted in reductions of drive for thinness, bulimic symptoms, and body dissatisfaction, superior to improvements seen in a cognitive behavioral therapy group and control group.

\section{Somatoform disorders}

The essential feature of these disorders is somatic symptoms which are not explained by a medical condition (MUS) and not better diagnosed as part of a depressive or anxiety disorder. Several syndromes are described within this category; there is considerable overlap between them in both aetiology and clinical presentation.

\section{Aetiology}

The cause of somatoform disorders is incompletely understood but contributory factors include depression or anxiety, the erro- neous interpretation of somatic symptoms as evidence of disease, and excessive concern with physical illness. A family history or previous history of a particular condition may have shaped the patient's beliefs about illness. Doctors may exacerbate the problem, either by dismissing the complaints as non-existent or by overemphasising the possibility of disease.

\section{Somatisation disorder}

Somatisation disorder (Briquet's syndrome) is characterized by the occurrence of chronic multiple somatic symptoms for which there is no physical cause. Symptoms start in early adult life and may be referred to any part of the body. It is much more common in women. Common complaints include pain, vomiting, nausea, headache, dizziness, menstrual irregularities and sexual dysfunction. Patients may undergo a multitude of negative investigations and unhelpful operations, particularly hysterectomy and cholecystectomy. There is no proven treatment but minimization of iatrogenic harm from investigation and attempts at medical treatment is important.

\section{Hypochondriacal disorder}

Patients with hypochondriasis have a strong fear or belief that they have a serious, often fatal, disease that persists despite appropriate medical reassurance.

They are typically anxious and seek many medical opinions and investigations in futile but repeated attempts to relieve their fears. CBT may be helpful. The condition may become chronic. In a small proportion of cases, the conviction that disease is present reaches delusional intensity, the best-known example being that of parasitic infestation ('delusional parasitosis'), which leads patients to consult dermatologists. Antipsychotic medication may be effective.

\section{Body dysmorphic disorder}

This describes a preoccupation with bodily shape or appearance, with the belief that one is disfigured in some way (previously known as dysmorphophobia). People with this condition may make inappropriate requests for cosmetic surgery. CBT may be helpful. The belief in disfigurement may sometimes be delusional, in which case antipsychotic drugs may help.

\section{Somatoform autonomic dysfunction}

This describes somatic symptoms referable to bodily organs which are largely under the control of the autonomic nervous system. The most common examples involve the cardiovascular system (cardiac neurosis), respiratory system (psychogenic hyperventilation) and gut (psychogenic vomiting and irritable bowel syndrome). Antidepressant drugs and CBT may be helpful. 


\section{Somatoform pain disorder}

This describes severe, persistent pain which cannot be explained by a medical condition. Antidepressant drugs (especially tricyclics and dual action drugs such as duloxetine and mirtazapine) are helpful, as are some of the anticonvulsant drugs, particularly carbamazepine and gabapentin. CBT and multidisciplinary pain management teams are also useful.

\section{Chronic fatigue syndrome}

Neurasthenia is characterised by excessive fatigue after minimal physical or mental exertion, poor concentration, dizziness, muscular aches and sleep disturbance.

This pattern of symptoms may follow a viral infection such as infectious mononucleosis, influenza or hepatitis. Symptoms overlap considerably with those of depression and anxiety.

There is evidence that many patients improve with carefully graded exercise and with CBT.

\section{Dissociative (conversion) disorder}

This has replaced the term 'hysteria' in the ICD-10 classification. It is characterised by a loss or distortion of neurological function not fully explained by organic disease.

The most common symptoms mimic lesions in the motor or sensory nervous system.

Dissociative disorder can also involve psychological functions, especially memory and general intelligence. The aetiology of dissociation is unknown. It has been considered to be the result of unconscious psychological processes and there is an association with adverse childhood experiences, including physical and sexual abuse. Organic disease may facilitate dissociative mechanisms and provide a model for symptoms; thus, for example, non-epileptic seizures may occur in those with epilepsy. Coexisting depression should be treated with CBT or antidepressant drugs.

The symptoms of patients with conversion disorder can be debilitating and include paralysis of one or more limbs, ataxia, tremors, tics, and dystonia. Other terms used to describe this disorder are functional gait disorder, hysterical paralysis, psychosomatic disorder, conversion reaction, and chronic neurosis. A common motor complaint is astasia-abasia, which is an unsteadiness of gait presenting with unusual in coordination especially in walking or standing still.General management of patients with medically unexplained symptoms is based on general principles and specific measures for individual syndromes.

\section{Reassurance}

Patients should be asked what they are most worried about. Clearly, it may be unwise to state categorically that the patient has no disease but it can be emphasized that the probability of having disease is low. If patients repeatedly ask for reassurance about the same issue, they may have hypochondriasis.

\section{Explanation}

Patients need a positive explanation for their symptoms. It is unhelpful to say that symptoms are psychological or 'all in the mind', but useful to describe a plausible physiological mechanism for the symptom that emphasizes the link with psychological factors such as stress and which demonstrates that the symptoms are reversible. For example, in irritable bowel syndrome, psychological stress results in increased activation of the autonomic nervous system which leads to constriction of smooth muscle in the gut wall, which in turn causes pain.

\section{Advice}

This should focus on how to overcome probable perpetuating factors: for example, by resolving stressful social problems or by practising relaxation. The doctor can offer to review progress, to prescribe (for example) an antidepressant drug and, if appropriate, to refer for physiotherapy or psychological treatments. The attitudes of relatives may need to be addressed if they have adopted an over-protective role, unwittingly reinforcing the patient's disability.

\section{Drug treatment}

Antidepressant drugs are often helpful, even if the patient is not depressed.

\section{Psychological treatment}

There is moderate evidence for the effectiveness of CBT. Other psychological treatments may also have a role. Learning theory and behavioral shaping formed the basis for the behavioral modification treatment approach. The rationale behind this treatment approach is that the conversion disorder symptoms are learned maladaptive behaviors. The patients believe that they have a particular neurological condition. They often assume a "sick role" with its privileged social status and rewards of attention. A vicious cycle develops in which the patients believe that they are sick and then receive reinforcement of this sickness through the rewards and attention of others. The treatment goal of behavioral modification is to reduce unwanted behaviors and strengthen desired behaviors [1-15]. 


\section{Physiotherapist role}

1. One of the main goals of rehabilitation of the patient with conversion disorder is to assist the individual to move away from a "sick role" and to return to healthy roles in their social, physical, and work life. There are numerous reports describing the need for physical therapy in the successful treatment of conversion disorder. However, there is a lack of reports that describe specific physical therapy intervention techniques or program progression.

2. The therapy management program used a behavioral modification approach to support classic physical therapy techniques of strengthening, gait training, neuromuscular reeducation, and balance training.

3. The basic Skinnerian learning theory proposes that consequences govern human behavior. If a unit of behavior produces a favorable reaction (reinforcement), there is an increased probability that the behavior will occur again. If the behavior does not provide reinforcement, it will be discontinued. The unwanted behaviors (abnormal gait, ataxia, and paralysis) were not punished but were ignored, and the desired behaviors (normal gait, smooth movements, and normal strength) were rewarded. The therapist asked the patient to stop and sit down and rest if an abnormal gait pattern or behavior occurred. Verbal cues included "take a break" and "regain control of your body." The therapist did not comment about the abnormal gait pattern. Instead, when the patient demonstrated the correct gait or movement pattern, the therapist provided positive reinforcement.

4. Treatment Progression- Develop rapport, Initial evaluation and mutual goal setting with staff, patient, and family. Patient involvement in selection of rewards/privileges Consistent therapy, physician, and nursing staff.

- Gaining trust of the patient

- Pregait activities stretching, general strengthening, bed mobility skills

- Sitting and standing balance activities

- Coordination activities

- $\quad$ Transfer training

- Weight-shifting activities

- Supported gait activities standing and beginning gait training in parallel bars or walker

- Gait broken down into stepping and weight shifting

- Progression to step to and then step over step gait pattern

- General mobility Progression to gait outside of parallel bars or walker

- Maneuvering around obstacles

- Increasing endurance, add bike, treadmill
- Privilege of walking in room and to therapy sessions

- Multitasking activities such as walking and talking, basketball dribbling, etc.

- Community reintegration Walking outside of therapy and hospital setting

- Architectural barriers incorporate job/school/homemaking tasks

- Activities in the community

- Discharge planning with family

Treatment programs for analogous neurological conditions (hemiparesis, paraplegia, quadriplegia, and ataxia) form the basis for the therapy treatment program. The patient progresses through the treatment program as if he or she had an organic neurological syndrome.

Treatment progresses through stages of recovery with each stage building on the previous stage.

The progression to the next stage does not occur until complete mastery of the previous stage.

Emphasis is placed on the quality of movement instead of the quantity of movement.

The patient receives verbal cueing to gain control of his or her extremities and focuses on the quality of the movement instead of the speed or distance. Developing rapport and trust with the patient is essential. Treatment should occur in a positive, nonthreatening environment. The patient is involved in goal setting. A contract toward goals can be helpful. Abnormal movements are ignored. All attention to illness symptoms is withdrawn. Assistive devices are removed as soon as possible

Implications for clinicians who wish to apply exercise

- $\quad$ First, to prevent potential ethical and legal conflicts, clinical psychologists who have not received training in the principles of exercise physiology might seek interdisciplinary liaisons with physicians and exercise physiologists. At the very least, clinicians should familiarize themselves with the guidelines for exercise testing and prescription set out by the American College of Sports Medicine. The benefits of such efforts, particularly as further evidence of the clinical effectiveness of exercise therapy is documented, are a broadening of practice possibilities and a possibly expanding base of potential referrals

- Second, medical clearance should always be obtained prior to commencing any formal exercise program. King and Senn outlined a useful framework for categorizing risk factors. 
- Class 1 individuals are those whose risk factor profile shows them to be apparently healthy, and they require only physician clearance to commence an exercise program.

- Class 2 individuals are those whose risk assessment shows them to be at higher risk for complications, and they require a limited symptom graded exercise test prior to receiving clearance to exercise.

- Class 3 individuals are known to have cardiac, pulmonary, or metabolic disease, and they are required to undergo graded exercise testing, pass more stringent criteria, and be more closely monitored by medical personnel.

Third, clinicians should consider the following useful and timely guidelines provided by Sime for the clinical application of exercise therapy:

1. Explore the client's exercise history to determine current exercise habits and experiences in order to identify enjoyable activities critical to program adherence.

2. Participate in initial exercise sessions to serve as a model for appropriate client behavior.

3. Educate the client about the potential physical and mental health benefits of exercise as a commitment enhancement procedure.

4. Consider options to make exercise functional, such as commuting to work by walking, jogging, or biking or including home chores in the prescription.

5. Take advantage of the client's environment (e.g., parks, lakes, fitness trails, home equipment) in facilitating exercise activity.

6. Help the client choose enjoyable activities from a broad spectrum of choices.

7. Prescribe the type, duration, frequency, and intensity of the exercise program in terms of the client's current level of conditioning. Clinicians who are not trained or experienced in exercise physiology are advised to seek the assistance of a local specialist who can supervise the ongoing prescription process.

8. Attempt to facilitate exercise within a positive social milieu.

9. Assist the client to develop behavioral self-control strategies (e.g., behavioral contracting, stimulus control, positive reinforcement) to improve program adherence.

10. Prepare the client for recidivism and reinitiating using relapse prevention strategies.
Drugs used and their side effects.

Anti Psychotics: dizziness, drowsiness, parkinsonism, postural hypotension, tardive dyskinesia. sensitivity to uv light.

- Lithiyum carbonate: Fatigue, ataxia, tremor, hyper reflexia (exs may alter the serum levels)

- Anxiolytics benzodiazepines: Drowsiness, poor coordination, impaired concentration, tachycardia, tremors.

- Hypnotics: Rebound insomnia, morning hangover.

- Anti depressents: Postural hypotension, drowsiness, dizziness, fatigue.

- Anticonvulsants: Dizziness, drowsiness, fatigue, ataxia, tremor.

\section{Conclusion}

Therapy for Psychiatric Disorders Considering the reviewed evidences are promising. Exercise is a viable, cost-effective treatment for depression and chronic pain and that it shows promise for other disorders. It is somewhat surprising that it has not become a more popular treatment alternative. The mechanisms responsible for exercise-related improvements in depression and anxiety disorders are not all known, and it is most likely to be a complex interaction of psychological and neurobiological mechanisms underlying, mediating and/or moderating these effects. Now we are far away from a conclusive model explaining the antidepressant and anxiolytic activity of exercise.

\section{Bibliography}

1. Davidson's principles and practice of medicine.

2. Cash's textbook of general medical and surgical conditions for physiotherapist.

3. Richard A Brown., et al. "A pilot study of aerobic exercise as an adjunctive treatment for drug dependence". Mental Health and Physical Activity 3 (2010): 27e34.

4. TW., et al. "Exercise therapy, cardiorespiratory fitness". European Neuropsychopharmacology (2012),

5. Lawrence S., et al. "Sports and games for post-traumatic stress disorder (PTSD) (Review)". The Cochrane Collaboration.

6. Peter Salmon. "Effects of physical exercise on Anxiety, depression, and sensitivity To stress: a unifying theory". Clinical psychology review 21.1 (2001): 33-61.

7. Debra Ness. "Physical Therapy Management for Conversion Disorder: Case Series". JNPT 31 (2007): 30-39. 
8. L Dratcu. "Physical exercise: an adjunctive treatment for panic disorder? CASE REPORT". European Psychiatry 16 (2011): 372-374.

9. Haffmans PMJ., et al. "Comparing running therapy with physiotraining therapy in the treatment of mood disorders". Acta Neuropsychiatrica 18 (2006): 173-176.

10. Gregg A., et al. "Exercise Therapy for Patients with Psychiatric Disorders: Research and Clinical Implications". Professional Psychology: Research and Practice 30 (1999): 275-282.

11. A.M. Abrantes., et al. "Exercise preferences of patients in substance abuse treatment". Mental Health and Physical Activity 4 (2011): 79-87.

12. Nicola T., et al. "The influence of exercise on brain aging and dementia". Biochimica et Biophysica Acta 1822 (2012): 474481.

13. Noriatsu Tatematsu., et al. "The effects of exercise therapy on delirium in cancer patients: a retrospective study". Support Care Cancer 19 (2011): 765-770.

14. Ivanka Prichard., et al. "Relations among exercise type, selfobjectification, and body image in the fitness centre environment: The role of reasons for exercise". Psychology of Sport and Exercise 9 (2008): 855-866.

15. Andreas Strohle. "Physical activity, exercise, depression and anxiety disorders". Journal of Neural Transmission 116 (2009): 777-784.

Volume 2 Issue 7 July 2019

(C) All rights are reserved by Pradeep Thotekat. 